\title{
Bioanalytical method validation: notable points in the 2009 draft EMA Guideline and differences with the 200I FDA Guidance
}

\author{
"Contrary to earlier expectations, much of the content of the European Medicines Agency draft Guideline actually closely \\ follows the US FDA Guidance and the subsequent American Association of Pharmaceutical Scientists publications."
}

The current US FDA Guidance for Industry on Bioanalytical Method Validation was published in May 2001 [101]. This guidance is almost universally adopted by the pharmaceutical and contract research industries as the standard approach for validating and implementing bioanalytical methods that are used to generate data to support medicinal product applications. The European Medicines Agency (EMA) does not currently have a guidance on bioanalytical method validation; therefore it published a concept paper on this topic in December 2008 as a discussion document [102]. Following a period for review and comment, the EMA then published a draft Guideline on the Validation of Bioanalytical Methods in November 2009 [103]. The end of the consultation period for the draft document is 31 May 2010 and it is likely that the Guideline will be finalized within the following 6 months. Therefore, it is important for laboratories working in this particular field to understand the main differences between the FDA and the EMA guidance documents.

The 2001 FDA Guidance for Industry is an official FDA publication. However, it is supplemented by numerous reports that were published following workshops co-sponsored by the FDA and the American Association of Pharmaceutical Scientists (AAPS) that were held after the original guidance document was issued. The workshop reports appeared in the AAPS Journal and were intended to clarify various points in the original guidance document and to address bioanalytical issues that had manifested themselves during the ensuing years [1,2]. Although the AAPS publications are not official FDA documents, they do reflect current working practices in the industry and therefore they have to be considered when comparing the 2001 FDA Guidance to the draft EMA Guidelines.
The main points to note and the key changes in the EMA draft Guideline (referred to hereafter as the 'Guideline') in relation to the current FDA Guidance are discussed where relevant, on a section-by-section basis.

\section{Section I: introduction (background)}

The Guideline makes it clear that there are certain circumstances when it may be necessary to use wider than normal acceptance criteria for bioanalytical methods, for example when analyzing complex matrices such as tissue samples. These types of sample can present a real analytical challenge for a number of reasons. For example, is spiking drug into a tissue homogenate representative of an intact sample and what is the best way to evaluate stability (e.g., in the intact sample or in the homogenate)? Often with complex matrices there may only be small numbers of samples and there is frequently more work involved in performing a method validation, which can be of questionable value in these circumstances. The approach described in the Guideline indicates the rational needs to be justified on a case-by-case basis and the acceptance criteria defined for each study. However, this is pragmatic advice for dealing with potentially difficult analytical situations that was not addressed in the FDA Guidance.

\section{Section 3: legal basis}

The main point to note in this section is that the Guideline addresses the compliance status of bioanalytical studies. This is something that none of the previous guidelines have dealt with satisfactorily, as it clarifies the distinction between preclinical and clinical studies. The Guideline states that bioanalytical method validations and the subsequent sample analysis should be carried out in compliance with GLP.

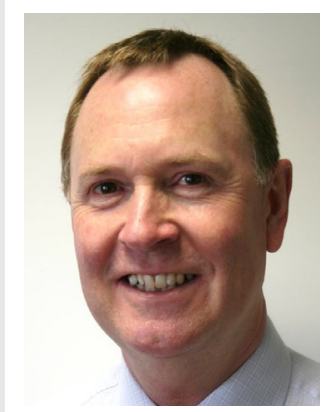

\section{Graeme Smith}

Scientific Director, Department of Bioanalysis, Huntingdon Life Sciences, Woolley Road, Alconbury, Huntingdon, Cambridgeshire, PE28 4HS, UK

Tel.: +44 1480892840

Fax: +44 I48 089228 ।

E-mail: smithg@

ukorg.huntingdon.com 
However, it highlights the fact that human bioanalytical studies fall outside the scope of GLP and that the analysis of samples from clinical trials should be carried out in conjunction with good clinical practice. The Guideline, however, does not sufficiently clarify the compliance status of method validations used to support clinical studies. It implies that these validations should be carried out in compliance with GLP, when this is not the case, as the work is being carried to support clinical studies. Therefore, the Guideline needs to be made clearer to address this grey area. Currently, the only claim that can be made for these particular validations by laboratories that are members of their national GLP compliance program is that the study was "conducted in laboratories that operate in compliance with GLP".

Overall, the Guideline is more definitive about addressing the compliance status of bioanalytical studies than the FDA Guidance.

\section{Section 4.I: complete validation of an analytical method}

If a bioanalytical method is fully validated in a particular preclinical species, the extent of the validation required in a second or third species has been an area of some debate. Indeed, the Partial Validation section of the 2001 FDA Guidance document states that if the species changes, only a partial validation of the method is required in the new matrix. In some cases, this has resulted in a reduction of the validation experiments carried out. The Guideline is unequivocal on this matter and states that a full validation of the method should be carried out for each different species.

\section{- Reference standards}

Both the FDA and the EMA documents deal with reference standards of the test compound in a similar way, with respect to the origin of the material and documentation requirements (e.g., certificate of analysis to identify the test compound, cover storage conditions, expiry dates, batch numbers and purity). Both documents also make it clear that certificates of analysis are not required for internal standards, but it is necessary to demonstrate that there are no impurities present in the internal standard that may interfere with the measurement of the test compound.

The Guideline takes this recommendation further and states that the suitability for use of the reference standard (test compound) needs to be scientifically justified. It also states that the use of stable isotopically labeled internal standards are recommended for methods that use MS detection. These need to be labeled in such a way as to minimize isotope-exchange reactions with the unlabeled test compound, and the isotope purity needs to be as high as possible in order to reduce any interference with measurement of the test compound by unlabeled internal standard.

\section{Section 4.I.I: selectivity}

The back-conversion of labile metabolites to the test compound is a key point that is discussed in this section that was not addressed in such detail in the FDA Guidance. The Guideline recommends spiking blank matrix (and blank matrix containing the parent drug spiked at a concentration approximately three-times higher than the lower level of quantification) with levels of metabolite at high in vivo concentrations. The spiked control matrix samples are then taken through the sample-preparation procedure and any back-conversion of the metabolites to the test compound is monitored. It is acknowledged that this may not be possible in the early development stages of a new chemical entity (NCE), but it is something that needs to be addressed as the development of the drug progresses.

\section{Section 4.I.2: carry-over}

Carry-over was not mentioned per se in the 2001

FDA Guidance, but it was dealt with in the 2007

White Paper [1]. The FDA and the EMA both take a similar line on carry-over, in that it needs to be evaluated and minimized during method development and validation and carefully controlled thereafter. For this reason, the EMA go a step further than the FDA and recommend that, for chromatography methods, the randomization of injection of samples should be avoided as this process may make it harder to detect and resolve carry-over problems.

\section{Sections 4.I.3 \& 4.I.4: lower limit of} quantitation \& calibration curve

With regard to describing the lowest measurements that can be made on a calibration curve, the FDA use the term 'quantification' (e.g., lower level of quantification) whilst the EMA have now introduced the term 'quantitation' in the same context. Although this may seem to be a minor point, some thought should be given to standardization of this and other essential terminology for describing pivotal analytical parameters, in order to avoid confusion. 
The 2001 FDA Guidance and the 2007 White Paper both discuss the use of calibration curve ranges that are appropriate to the concentration of test compound that is likely to be measured in study samples. The Guideline reiterates this point by stating that the lower level of quantification for the method should be adapted to meet the expected concentrations of drug in samples and that before the method validation is carried out, it should be known what concentration range is expected. This is a logical position to take, but it must be remembered that information regarding matrix concentrations of NCEs in early development may not be known when the first preclinical methods are validated.

The EMA discuss the acceptability of calibration curves and their proposals are in line with what the FDA recommends. One other important point to note in the Guideline is the recommendation that freshly prepared calibration standards are used during validation, even if stability data indicate that the test compound is stable in the matrix. This point may impact on how some laboratories carry out their method validations, because the approach of using bulk prepared matrix calibration standards at the start of the study that are then frozen and then checked for stability at the end of the study is quite a common practice.

\section{- Sections 4.I.5 \& 4.I.6: accuracy (incorporating within- \& between-run accuracy) \& precision}

There has always been some debate in this field about where to place the high QC sample in relation to the upper boundary of the calibration curve. Some advocate that during validation the QC should be placed at the ULOQ in order to be able to evaluate the precision and accuracy at the highest point of the calibration curve. Critics argue that this approach inevitably means using some data that have to be calculated by extrapolation and, consequently, a QC that is set at the ULOQ during validation is at too high a concentration to be used during subsequent sample analysis. Therefore, a more pragmatic view is to set the high QC at a level below the ULOQ so that values within $\pm 15 \%$ of the nominal value of the $\mathrm{QC}$ are still within the upper boundary of the calibration curve. Using this approach, the high QC can be checked for accuracy of preparation during validation and then used to monitor method performance during sample analysis. The EMA recommend setting the high QC at $75 \%$ of the ULOQ, but this concentration is likely to be too low to evaluate precision and accuracy at the top of the calibration curve. The 2001 FDA Guidance is less specific and simply recommends placing the high QC "near the upper boundary of the standard curve". For chromatographic methods, the most appropriate level to set the High QC and still obtain acceptable precision and accuracy data is at or near to $85 \%$ of the ULOQ.

There are two other notable points made in this section by the EMA. First, the recommendation that at least one of the batches analyzed during validation should be approximately the same size as the anticipated batch size during sample analysis, so that any trends that develop in a batch over time can be evaluated. However, it should be remembered that during validation it may not always be possible to predict the size of future sample analysis batches. Does this mean that if batch sizes are subsequently increased then further validation experiments are required to support the larger batches? Or can a more pragmatic approach be taken? For example, during sample analysis, if the calibration standards are placed at the start and end of the batch and the QCs are spread throughout the batch and all the necessary acceptance criteria are met, then presumably this demonstrates that the batch size is valid, even if it is larger than the batches that were analyzed during validation.

The second point to note is that reported results for validation experiments (including accuracy and precision evaluation) should include all the data for a particular experiment (e.g., all replicate QC measurements). However, if the result for the measurement of an individual replicate of a QC is statistically shown to be an outlier then it can be removed and the calculations repeated. In these circumstances, the results of the calculations should be presented with and without the outlier.

With regards to between (inter)-batch accuracy and precision, neither the FDA nor the EMA have got to grips with the fact that most laboratories do not report true between-batch data. What typically happens is that a summary is produced of the combined accuracy and precision data for three analytical batches and a statistical summary is produced for the whole of the data set (e.g., mean, standard deviation and coefficient of variation). Consideration should therefore be given to using a more appropriate description of this approach such as "overall-batch accuracy and precision." 
- Section 4.I.8: matrix effect

The 2001 FDA Guidance only briefly mentions matrix effects, however the 2007 White Paper deals with this topic more comprehensively. The Guideline makes several additional recommendations for performing matrix-effect experiments that need to be noted.

The Guideline states that for MS methods, matrix effects should be evaluated using six different sources of matrix. If the matrix is plasma or serum then this should include hemolysed and hyperlipidemic samples and, if possible, sample matrix from special populations (e.g., renally impaired). It is assumed that this statement has been made with clinical studies in mind, as it not easy to obtain some of the more specialized matrices on a routine basis in order to support preclinical validations.

The Guideline goes on to specify the concentration at which the test compound should be spiked into blank matrix (maximum of three times the lower level of quantification) so that experiments can be carried out to calculate the matrix factor.

It also recommends an approach for evaluating matrix effects when using online sample preparation systems (e.g., six batches of matrix analyzed in triplicate with the test compound spiked in the matrix at three-times the lower level of quantification).

For intravenous dose formulations, the Guideline recommends that excipients in the formulations that are known to cause matrix effects should be evaluated for possible ion suppression or enhancement effects in the matrix used in the bioanalytical method, in addition to an evaluation of matrix effects carried out in blank matrix only. The matrix used in this evaluation should be obtained from subjects or animals administered with the excipient unless it has been shown that the excipient is not transformed in vivo, in which case it can be spiked directly into the blank matrix.

\section{- Section 4.I.9: stability}

The main points to note for this particular topic are that the EMA make a general statement that recommends that stability should be evaluated to cover every step during sample preparation, analysis and sample storage to ensure that the concentration of the test compound in samples remains within acceptable limits. It recommends that the stability of the test compound and internal standard in matrix is evaluated by analyzing at least triplicate replicates of QCs at low and high concentrations immediately after preparation and following retention under the appropriate storage conditions. Following storage, the QCs should be analyzed against freshly prepared calibration standards and the measured concentration of test compound compared with the nominal concentration at which the QC was prepared (not the concentration measured at time zero). For freeze-thaw experiments, it is recommended that for each cycle, the QCs should be frozen for at least $12 \mathrm{~h}$ at the appropriate temperature. The acceptance criterion for stability experiments is formally documented for the first time and states that measurements must be within $\pm 15 \%$ of the nominal value at which the QCs were prepared.

Although the Guideline advocates evaluating the stability of the internal standard in matrix under similar storage conditions to the test compound, this is assumed to be a mistake.

The Guideline emphasizes that, although data in scientific publications may indicate that a test compound is stable in a particular matrix under a given set of conditions, it is still necessary to generate the appropriate stability data in the laboratory where the bioanalytical work is being conducted.

The recommendation that incurred samples should not be used for the evaluation of long-term stability is more contentious. The reason given is that the nominal concentration of test compound in the sample is unknown. However, carefully designed experiments using incurred samples can provide invaluable data on test-compound stability in the matrix. An initial analysis of the concentration of the test compound in the sample can be used as a starting reference point and then further measurements can be made at predetermined time points and the change in concentration of the test compound monitored over a of a period of time. The use of incurred samples is less artificial than using spiked QCs because the incurred samples will contain all the metabolites of the test compound, which are unlikely be present in spiked QCs. This approach may provide useful additional information about the storage of samples that would not be obtained using spiked QCs, particularly if some of the metabolites are unstable and degrade back to the test compound.

The Guideline also emphasizes the importance of addressing test-compound stability in the matrix during sample collection and during any necessary handling procedures prior to storage.

\section{Section 4.3: cross-validation}

Cross-validation is probably one of the most inconsistently used terms in bioanalysis and again emphasizes the need for harmonization 
of terminology in advisory guidelines in order to avoid confusion. In the 2001 FDA Guidance, cross-validation is defined as "a comparison of validation parameters when two or more bioanalytical methods are used to generate data within the same study or across different studies." This covers the analysis of samples from a single study on multiple sites or the use of different analytical techniques for different studies to support the same regulatory submission. However, in the 2007 White Paper, the term cross-validation is used in a different context in order to describe a series of validation issues (e.g., 'cross-validation' of methods when using different anticoagulant counter ions or matrices from different strains or species), when these would have been more appropriately covered by the term 'partial validation' as defined in the 2001 FDA Guidance.

The perspective that the Guideline takes on cross-validation is that it requires a comparison of analytical methods to be carried out when data are obtained from different study sites. This is to cover the use of the same method on different sites, modifications to that method (e.g., changes to the sample preparation procedure) or the use of a different type of method on different sites. The Guideline recommends that the cross-validation is carried out before the study samples are analyzed and advises that the same set of QCs should be analyzed using both methods. The acceptance criterion is that the difference between the two methods should not be greater than $15 \%$. The evaluation between the different methods is based solely on the use of the analysis of spiked QC samples. If they are available, consideration should also be given to using a proportion of incurred samples to evaluate the performance of different methods in these particular circumstances, as the use of test samples are more likely to highlight differences in the methods in a real situation.

\section{- Section 4.4: ligand-binding assays}

The section in the Guideline on ligand-binding assays seems to have been added as an afterthought and requires improving. There is no indication for these types of assays that it is normal to analyze all calibration standards, QCs and study samples in duplicate in order to compensate for assay variability.

It is recommended that specificity is checked by using at least 10 sources of matrix spiked with the test compound at or near the LLOQ. This may be difficult to achieve for preclinical methods owing to difficulties in obtaining matrix from a large number of different animals.
Precision and accuracy batches should include QCs at the LLOQ and the ULOQ as well as at the low, medium and high level. The current text is not clear on this point. Dilution, parallelism and prozone effect are also not covered in the Guideline.

The Guideline recognizes that the current main application of ligand-binding assays is for the measurement of macromolecules. Because the macromolecule in development may be similar to endogenous compounds, the interference in blank (control) matrix may exceed $20 \%$ of the LLOQ of the method. However, the Guideline states that this may be acceptable as long as it does not affect accuracy. More details are required on the validation of test compounds that are similar to endogenous molecules.

It is also recommended to run validation batches of similar size to the batch sizes anticipated during sample analysis and, if a batch comprises of several microtitre plates, then calibration standards and QCs should be placed on each plate to compensate for differences in performance.

If commercial kits are used to support applications for which they were not originally developed (e.g., a diagnostic kit used to support a bioequivalence study), then the kit must be validated to the necessary standard to suit its intended application.

\section{Section 5: analysis of study samples}

In the introduction to this section the point to note in the Guideline is that if a period of time has elapsed between method validation and sample analysis, it may be necessary to check the performance of the method again before any samples are analyzed. However, the Guideline does not offer advice on what this time period is or whether other factors (e.g., equipment reconfiguration, multiple methods running on a single instrument) also need to be considered when deciding to reverify the performance of a method.

\section{- Section 5.I: analytical run}

The Guideline defines the composition of an analytical batch (e.g., the batch should contain matrix blanks, a zero sample, calibration standards, QCs and study samples) and it recommends that the batch should be processed and analyzed as an integral unit. The Guideline states that analyzing a single run of samples prepared in several batches should be avoided (this is taken to mean, for example, a set of samples extracted in multiple 96-well plates but analyzed as a single batch). If the single run-multiple batch scenario cannot be avoided due to factors such as stability 
then QCs should be included with each batch (plate) and standard operating procedures should be in place to cover this approach.

It is recommended that, in order to reduce variability, all samples from a single subject should be run in the same batch and that it is acceptable to analyze samples from multiple subjects together. Advice is also given on the placement of QCs in the batch, however, this seems to be contradictory. Dividing the QCs throughout the batch is logical and provides information on the performance of the method throughout the run. However, suggesting that one of each of the low, medium and high QCs can be placed at the start of the batch and then again at the end of the batch is less useful as this approach provides no information about what is happening in the middle part of the batch during analysis.

\section{- Section 5.2: acceptance criteria of an analytical run}

The Guideline highlights the fact that that if the LLOQ or ULOQ calibration standards are rejected, then an abbreviated calibration range can still be used. The next acceptable calibration standard on the calibration curve then becomes either the revised LLOQ or ULOQ, depending on which standard is lost. Acceptability of this approach is also dependent on the revised calibration range covering all the QC samples.

When discussing acceptability of ligandbinding assays, the draft Guideline mentions nothing about analyzing calibration standards or test samples in duplicate.

\section{Section 5.3: calibration range}

The Guideline emphasizes the importance of the method having a calibration range appropriate to the concentrations of the test compound in the study samples and that the QCs should also cover the concentration of test compound in the samples. This point is not covered in the 2001 FDA Guidance, but it is dealt with in the 2007 White Paper.

\section{- Section 5.4: reanalysis of study samples}

The take-home messages in this section of the Guideline are that under normal circumstances the reanalysis of study samples for pharmacokinetic reasons is not acceptable. Similarly, the re-injection of batches of samples in which the calibration standards or the QCs have failed to meet the acceptance criteria is unacceptable, if the analytical problem that caused the failure has not been identified.

\section{Section 6: incurred samples reanalysis}

Incurred samples reanalysis (ISR) is not mentioned in the 2001 FDA Guidance, but the concept was discussed in the 2007 White Paper. However, it was not until the 2009 AAPS Workshop Report appeared, that detailed recommendations about running an ISR program and on appropriate acceptance criteria were published [2]. The Guideline deals with ISR as an integral part of its overall recommendations. The rationale and approach outlined for performing ISR is very similar to that described in the 2009 AAPS Workshop Report, as is the acceptance criteria. The main difference in the Guideline is that for preclinical studies it does not advise on how many samples should be reanalyzed. Nor does it offer advice on this matter for clinical studies, but it does state that where an ISR evaluation is conducted, incurred samples should be reanalyzed from each subject close to the expected maximum concentration of the test compound and in the elimination phase. This could potentially involve analyzing more samples than recommended in the 2009 AAPS Workshop Report.

\section{Section 7: study report}

The Guideline provides a list of detailed recommendations about the content of bioanalytical reports. The following points are recommendations made by the EMA that do not appear in the FDA Guidance:

\section{- Method validation}

- The report should include the study protocol and SOPs for relevant analytical procedures should be appended to the study report;

- Individual data should be available in electronic format;

- The report should include preparation dates of calibration standards and QCs and sample tracking information (conditions and duration of storage). The latter point presumably refers to storage of QCs.

\section{Sample analysis}

- The report should include sample-tracking information (including dates of receipt and storage location);

- Chromatograms from complete batches (including calibration standards and QCs) for $5-20 \%$ of subjects should be included in the report; 
- For bioequivalence studies, chromatograms from at least $20 \%$ of subjects are required.

\section{Summary}

When the EMA first announced its intention to publish a guideline to cover bioanalytical methods, many scientists working in this field thought that this was going to make their lives more complicated. What the pharmaceutical and contract research industries did not want from the EMA was a document that led to contradictions with the well-established FDA Guidance. Contrary to earlier expectations, much of the content of the EMA draft Guideline actually closely follows the FDA Guidance and the subsequent AAPS publications. For example, many of the recommendations from the EMA about running calibration standards and QCs and the associated acceptance criteria are very similar to what is in the FDA Guidance. Many of the differences that have been highlighted in this commentary article have arisen mainly because the approach taken in bioanalysis has moved on since the 2001 FDA document and the AAPS meeting reports were published.

In addition to some of the comments mentioned earlier, the EMA also need to look carefully at the terminology that they use as this should be harmonized with the FDA. They also need to review the ligand-binding assay section of the Guideline to provide more comprehensive advice to cover this particular field.

One of the major benefits of the EMA Guideline is that it pulls together into a single document all the key parameters that need to be considered when working with bioanalytical methods. If the final version of the Guideline is issued on the basis of comments received by the EMA following the issue of the Concept Paper, the draft Guideline and the European Bioanalysis Forum and European Federation for Pharmaceutical Sciences workshop (held in Brussels in April 2010), then hopefully this will be an all-encompassing document that accurately reflects how modern bioanalysis should be carried out within the industry. However, the ultimate goal remains - to have a single set of global guidelines that can hopefully cover the requirements of both the EMA and the FDA.

\section{Financial \& competing interests disclosure}

The author has no relevant affiliations or financial involvement with any organization or entity with a financial interest in or financial conflict with the subject matter or materials discussed in the manuscript. This includes employment, consultancies, honoraria, stock ownership or options, expert testimony, grants or patents received or pending, or royalties.

No writing assistance was utilized in the production of this manuscript.

\section{Bibliography}

1 Viswanathan CT, Bansal S, Booth B et al. Quantitative bioanalytical methods validation and implementation: best practices for chromatographic and ligand binding assays. AAPS J. 9(1), E30-E42 (2007).

2 Fast DM, Kelley M, Viswanathan C et al. AAPS workshop on current topics in GLP bioanalysis: assay reproducibility for incurred samples - implications of crystal city recommendations. AAPS J. 11(2), 238-241 (2009).

\section{- Websites}

101 US FDA. Guidance for Industry: Bioanalytical Method Validation. US Department of Health and Human Services, FDA, Center for Drug Evaluation and Research, Rockville, MD, USA (2001) www.fda.gov/downloads/drugs/ guidancecomplianceregulatoryinformation/ guidances/ucm070107.pdf
102 European Medicines Agency. Concept paper/ recommendations on the need for a (CHMP) guideline on the validation of bioanalytical methods. EMEA/CHMP/ EWP/531305/2008 (2008) www.emea.europa.eu/pdfs/human/ ewp/53130508en.pdf

103 European Medicines Agency. Draft Guideline on Validation of Bioanalytical Methods. EMEA/CHMP/EWP/192217/2009 (2009) www.ema.europa.eu/pdfs/human/ ewp/19221709en.pdf 